Eder, 'the tower of the flock' . . . where shepherds watched the templeflocks all the year round.

Edersheim bietet ja überhaupt eine ganze Reihe treffender jüdischer Seitenstücke zur Kindheitsgeschichte, die jedem Einsichtigen klar machen müssen, daß dieselbe aus jüdischen Gedanken herausgesponnen ist. Leider hat Edersheim diese Parallelen nicht zu nützen verstanden, wie noch so manche andere, die er aus dem jüdischen Schrifttum beigebracht hat, so z. B. die hochwichtigen zu Mt 16, 18. Warum sich die kritische Wissenschaft seinen umfassenden Kommentar aus der jüdischen Schrift nicht melir zu nutze macht, weiß ich nicht. Vielleicht liegt es an der Dickleibigkeit seines Werkes und dem Mangel an einer deutschen Übersetzung.

Basel.

K. G. Goetz.

\title{
Eine Vierteilung des neutestamentlichen Kanons.
}

Nachstehender Eingang des im Jahr I 158 verfaßten Katalogs der Büchersammlung des Bayrischen Klosters Prüfening verdient bekannter $\mathrm{zu}$ werden, als er es zu sein scheint.

Vt plene \& euidenter in noticiam ueniat inops armariae nostrae thesaurus quodam ordine uidetur procedendum. Libri alii sunt diuinae auctoritatis tam ueteris testamenti quam noui. alii humanae. De diuinis praemittendum \& sciendum quod sicut uetus testamentum quatuor ordinibus distinguitur, lege. prophetis. agyographis \& qui extra canonem sunt. Lex enim est in Ve libris moysi. Prophetarum sunt VIII. Iosue. Iudicum. Samuel. Malachim. Ysaias. Ieremias. Ezechiel. Liber XIIcim prophetarum. Agyographorum VIIII. Liber iob. Psalmorum. Proverbiorum. Ecclesiastes. Cantica cant'. Daniel. Paralypomenon. Esdras. Hester. Extra canonem sunt Tobias \& ivdith. nam liber sapientiae \& iesv filii Syrach nesciuntur apud hebreos. ita inquam quatuor ordinibus distinguitur \& nouum testamentum. Diuiditur enim in euangelia IIIIor in apostolos $/$ in apocalipsin. librum actuum apostolorum. canonicas epistolas \& epistolas beati pauli. in patres ./ de his omnibus vel de fide vel aliud quid ad edificationem scribentes. \& sunt extra canonem libri conditi praeter huiusmodi utilitatem. Itaque omnia ueteris testamenti. \& de nouo euangelia \& apostolos habemus in tribus ueteribus bybliothecis. Eosdem habemus in IIIIor nouis praeter psalterium \& euangelia. Seorsum tamen habemus psalterium quatuor editionum gallice 
Romanc Hebrayce grece in uno uolumine. seorsum libros salomonis \& librum iob. scorsum aplm seorsum canonicas epistolas et librum actum apostolorum.

Patres alii antiqui. alii moderni. Antiqui sunt etc.

Aus G. Becker, Catalogi Bibliothecarum antiqui 1885 p. 209. nach cod. Monac. 13002. Becker verwies auf $L$. Rockinger, Zum baicrischen Schriftwesen in: Abhandlungen der hist. Classe der k. b. Akademie XII (München 1874, 65) und Monumenta Boica vol. XIII (1777) 134-139. Die Stelle ist nicht ganz klar; die Zeichen im Text $\%$ scheinen selbst schon auf eine Textverderbnis hinzuweisen. Der alttestamentliche Teil geht natürlich auf Hieronymus zurück. Lehrreich, daff die Bezeichnung "Apostolus" sich bis ins 12. Jahrhundert gerettet hat.

Maulbronn.

Eb. Nestle.

\section{Salomo und Nathan in Mt I und Lc 3 .}

$\mathrm{DaB}$ der Stammbaum Jesu einerseits auf Salomo, andrerseits auf Nathan zurückgeführt wird, nimmt den nicht mehr wunder, der Zach I2, I2 im Targum nachsielt: „sie werden sehen, in welchen sie gestochen haben" ist im N. T. mehrfach auf Jesus angewandt, und V. I2 übersetzt das Targum: Es klagt die Nachkommenschaft des Königs Salomo vom Hause Davids ... und die Nachkommenschaft des Propheten Nathan des Solnes Davids ... und die Nachkommenschaft des Hauses Mardochai des Sohns Jair, des Sohns Simëi.

Hier haben wir von den vielen Söhnen Davids die beiden nebeneinander, auf die nun auch der Stamnibaum dessen zurückgeführt wird, in den sie gestochen haben. Im hebräischen Text ist nur vom Haus David und von dem Geschlecht „Nathans" die Rede; ob unter letzterem der Sohn Davids zu verstehen sei, streiten die Ausleger. Ob die Gleichsetzung des Propheten Nathan mit dem Sohne Davids auch sonst vorkommt, oder auf einer Gedankenlosigkeit des Targums beruht, habe ich nicht untersucht. Zur Deutung der ganzen Stelle von dem Messias dem Sohn Ephraims ist das Fragment des Jerusalemischen Targums zu vergleichen, das man am bequemsten in Lagardes prophetae chaldaice p. XL̇II liest. Auch in Volzs Jüdischer Eschatologie fehlt in dieser Hinsicht manches.

Maulbronn.

Eb. Nestle. 


\section{Zu Mt 2.}

Als ich in den Bibliographien unserer Zeitschriften las, daß Halévy in der Revue Sénitique 1903 in seinen Questions évangeliques auch les Rois Mages beliandelt habe, vermutete ich, ein jüdischer Schriftsteller vom Range Halévys werde ctwas Zutreffenderes zu dieser Erzählung beizutragen haben, als in neuster Zeit, auch in dieser Zeitschrift, beigebracht wurde. Denn, ehrlich gestanden, daß diese Erzählung durch die Huldigungsreise des Partherkönigs Tiridates nach Rom im Jahr 66 veranlaßt worden sein sollte, will mir trotz Dieterich (3, Iff.), Usener (4, 19) und andern äußerst unwahrscheinlich vorkommen, so wenig wie ich den hohen Berg der Versuchungsgeschichte so erklären möchte wie es in dieser Zeitschrift geschehen ist. Viel näher liegt es doch von jüdischem Boden auszugehen, und darum sah ich der Arbeit Halévys mit solcher Spannung entgegen. Um so größer war freilich meine Enttäuschung, als ich sỉe kennen lernte. In der Hauptsache läuft sein Bestreben darauf hinaus, die Kindheitsgeschichten bei Matthäus und Lukas miteinander auszugleichen. So erlaube ich mir selbst einige Beobachtungen mitzuteilen, die mir aufgestoßen sind, als ich vor kurzem erstmals das Matthäusevangelium statt wie bisher den. Markus mit meinen Schülern zu lesen anfing.

I. Längst erkannt ist, schon von Justin dem Märtyrer, wenn auch noch neustens von $\mathrm{Zahn}$ bestritten, die Beziehung von Mt 2 zu Num 23, 24; aber noch lange nicht alles ist aus der Bileamsgeschichte beigebracht, was die Magiergeschichte beleuchten kann. Daß die Heimat der Weisen

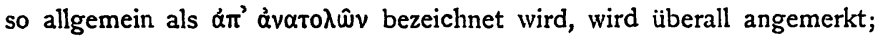
aber nur Holtzmann, soweit ich sehe, hat zu Mt 2, I die Stelle Num 23, 7 beigezogen. Auch Hühn-und Dittmar beginnen erst zu 2, 2 Num 24, 17 zu vergleichen. - Nun lese man nur

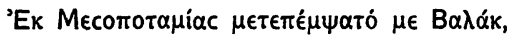

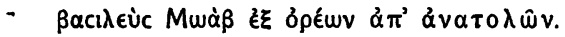

Hier haben wir sogar die Berge, auf denen nach der späteren Legende die Magier ihre Sternbeobachtungen angestellt haben. Die englische RV with marginal References heibt zu 'the East' Gen 25,6: I Reg 4, 30 vergleichen; ich meine, Num 23, 7 dürfte künftig in keinem Kommentar und keiner Ausgabe als Parallelstelle zu Mt 2, I fehlen. In Poles Synopsis sind die verschiedensten Ansichten aufgezählt, was unter dem Morgenland verstanden sein könnte. An erster Stelle wird aus Drusius 
angefuhrt: Forsan ex Mesopotamia, unde Balaam, ex cuius progenie fuisse crcduntur.

Dann finde ich nur noch bei Holtzmann: zumal Num 23, 7 kommit Bilcam, dessen Stern sofort leuchten soll, a $\pi$ àvatodûv.

2. Auch für den antiken Volksglauben, dab die Stcrne am Himmel die Geburt eines großen Mannes verkündigen, führt Usener IV, ig einige Beispiele an, selbst hinzufügend, dals dies semitischer Herkunft sein könne. Warum bleiben wir für den, der I, I der Sohn Davids des Sohns Abrahams genannt wird, nicht bei seinen Vorfahren? Wozu Alexander den Großen oder Alexander Severus beiziehen? Nun lese man nur einmal, wie Abrahans Geburt in der rabbinischen Literatur geschildert wird. In the Jewish Encyclopedia ist es folgenderma@en zusammengefabt $(I, 86)$ :

On the night when he was born, Terah's friends, among whom where councilors and soothsayers of Nimrod, were feasting in his house, and on leaving late at night they observed a star which swallowed up four other stars from the four sides of the heavens. They forthwith hastened to Nimrod and said: 'Of a certainty, a lad has been born, who is destined to conquer this world and the next; now, then, give to his parents as large a sum of money as they wish for the child, and then kill him'. But Terah, who wras present, said: 'Your advice reminds me of the mule to whom a man said, 'I will give thee a house full of barley if thou wilt allow me to cut off thy head', whereupon the mule replied: 'Fool that thou art, of what use will the barley be to me if thou cuttest off my head?' Thus I say to you: if you slay the son, who will inherit the money you give to the parents?' Then the rest of the councilors said: 'From the words we perceive, that a son has been born to thee'. 'Yes', said Terah, 'a son has been born to me, but he is dead'. Terah then went home and hid his son in a cave for three years.

Hier haben wir vier Momente der Erzählung beieinander: den Stern, die Wahrsager, den verfolgenden König, die Rettung des Kindes. Ich weiß nicht, ob sich das Alter dieser Abrahamsgeschichte ausmachen läßt; aber das dürfte klar sein, daß es ganz derselbe Boden, und zwar spezifisch jüdischer Boden ist, auf dem beide erwuchsen.

Das Vorstehende ist keine Lösung der Frage, woher der Stoff von Mt 2; aber auch der Hinweis, dạ man eher in der jüdischen Haggada als in heidnischen Anschauungen $z u$ suchen hat, wird nicht wertlos sein. Maulbronn.

Eb. Nestle. 


\section{Das ursprüngliche Neue Teștament nach W. Whiston.}

William Whiston, 1667-1752, U̇bersetzer des Josephus und Herausgeber eines Buchs Primitive Christianity Revived ließ im Jahr 1745 in $8^{\circ}$ auf seine Kosten drucken:

Mr. Whiston's Primitive New Testament. Part I-IV.

Der erste Teil, die Evangelien und Apostelgeschichte, folgt dem Kodex: Bezä und ergänzt dessen Lücken aus der Vulgata, der zweite, die vierzehn paulinischen Briefe, folgt dem Claromontanus, der Rest (Teil $3=7$ katholische Briefe, $4=$ Offenbarung) dem Alexandrinus. Ein Blatt am Schluß enthält folgende gedruckte Nachricht:

Mr. Whiston's Primitive New Testament. Part V containing the Epistle of the Corinthians to Paul, and his Answer, preserved by the Armenians. The Epistle of Timothy to Diognetus, and the Homily. With the two Epistles of Clement to the Corinthians. Part VI containing the Constitutions of the Apostles in VIII Books. Part VII containing the Catholick Epistle of Barnabas. With the Shepherd of Hermas, in III Books. Part VIII. containing the X Epistles of Ignatius. The Epistle of Polycarp to the Philippians. Josephus's Homily concerning Hades. With the martyrdom of Polycarp.

Der „Historical Catalogue of the Printed Editions of Holy Scripture in the Library of the British and Foreign Bible Society compiled by T. H. Darlow and H. F. Moule (London 1903), dem ich das Vorstehende entnehme, sagt dazu (I 276): es sei mehr als zweifelhaft, ob diese weiteren Teile des Werks je veröffentlicht wurden. Aber auch so wird diese Zusammenstellung, die das Programm dieser Zeitschrift vor mehr als 150 Jahren vorwegnahm, vielen Lesern willkommen sein. Sie gibt manches zu denken.

Maulbronn.

Eb. Nestle.

\section{Acta 27, r7.}

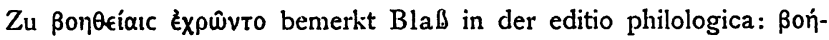
$\theta \in 1 \alpha$ (Hebr. 4,16 ) hic de eis consiliis quibus ... tutiorem navem reddere studebant. Sicherlich richtiger Wetstein: adjumenta quibus ad firmandam navem utebantur. Vollends wenn sich belegen lälit, was er hinzufügt: In scriptoribus mechanicis $\beta$ on $\theta$ eíai (so) vocantur, quae ad structuram collapsam sustentandam supponuntur, also "Stitzen". In der syrischen Bibel wird das hebräische תר ictóc, Mastbaum durch li wörtlich „Hilfe", Stütze wiedergegeben. In den Acta Thomae sagt der sich für einen 
Zinmmermann ausgebende Thomas auf die Fragc, was er machen könne: Pflüge und Joche und Ochsenstachel und Ruder für Fähren und it لإحس. Wright übersetzte masts for ships, fügte aber in einer Anmerkung hinzu, dab es ebensogut the large beans or girders of a ship bedeuten könnc (s. Wright, Apocryphal Acts II, p. $148=$ Bedjan, Acta Martyrum III, 5 letzte Zeile; 18, 7). Schon der Thesaurus Syriacus 2815 erinnert an Act 27, 17. Ich habe keinen $Z$ weifel, dab wir hier einen terminus technicus der Schiffer- und Handwerkersprache haben. Aus Philo kann ich noch anführen (IV, 68, 14 der neuen Ausgabe $=I I, 47 \mathrm{M}): \ddot{\omega} c \pi \epsilon \rho$

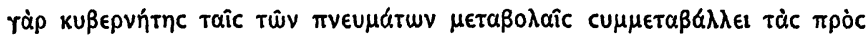

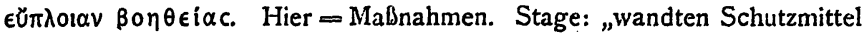
an“; ich würde übersetzen: „verwendeten Stützen“.

Maulbronn.

Eb. Nestle.

\section{- Der Magier in Josephus, Antiq. XX.}

(Zu Bd. V, S. 127 f.)

Alles was Hans Waitz über die Identität des Simon Magus der Apostelgeschichte mit dem „Magier Simon von dem Josephus Antiqu. XX, 7, 2 erzählt" geschrieben hat, wird durch den Hinweis von Harris hinfällig, daß. der Magier des Josephus gar nicht Simon, sondern nach

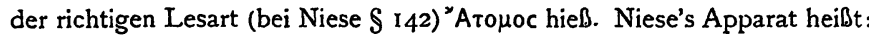

"Atouov] AE címwvoc MW Lat et i marg. A.

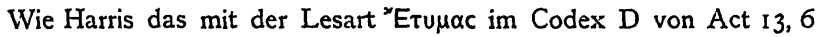
verbunden hat, sehe man bei ihm selbst nach (A curious Bezan reading vindicated: Expositor, March 1902. 189-195). In Deutschland hat Clemen in der $\mathrm{Th} \mathrm{Lz}_{2}$ 02, 325 auf die Arbeit von Harris aufmerksam gemacht, indem er den Druckfehler Ant. XX, 72 (statt 7,2 ) von Harris übernahm; ebenso in der Th Rds. 1903,83 , ebenso ganz ausführlich Knopf in dem (mir am 14. Okt. 1903 zugekommenen) neutestamentlichen Teil des Theol. Jahresberichtes für 1902 S. 307 f., mit Verweisung auf Clemen's zweite Äußerung. Endlich auch Th. Zahn (Zur Lebensgeschichte des Paulus in: NkZ XV, 3 S. I9of.). Letztere Stelle konnte in dem am 18. April 1904 abgeschlossenen Aufsatz von Waitz noch nicht wohl verwertet werden; aber durch einẹ der drei anderen Stellen hätte die Gleichsetzung des Atomos mit dem Hetoimos-Elymas bekannt werden können. $\mathrm{Z}$ ahn scheint unabhängig von Harris auf diese Vermutung gekommen zu sein, da er ihn nicht erwälint. Daß Waitz die richtige Lesart des 
Josephus gänzlich übersah, ist um so auffallender, als sie auch schon von Schmiedel mit Berufung auf Harris in der von Waitz (S. 127) zitierten Sp. 4550 seines Art. Simon Magus in der Encycl. Bibl. (1903) angeführt wurde.

Maulbronn.

Eb. Nestle.

\section{Der Schwur auf das Evangelium.}

Seit wann wird in der Kirche der Eid auf das Evangelium abgelegt? Das Opus. imperfectum in Matthaeum hat zur Bergpredigt einen eigenen Excurs Contra clericos qui Evangelia porrigunt juraturis. Er beginnt (Migne 56,698): Audite vos, clerici, qui jurantibus Evangelia sancta porrigitis: quomodo potestis ab illo iuramento esse securi, qui semen perjurii datis. Ein folgender Abschnitt steht nicht in allen Handschriften: Si erat bene jurare justum, juste dicebatis, quia dedimus illis Evangelium ut jurent, non ut perjurent; nunc autem cum sciatis, quia et bene jurare peccatum est, quomodo potestis esse liberi, qui occasionem datis unde peccetur in Deum.

Die Zeit des Opus imperfectum ist streitig; aber auch so wird dieser Beitrag zur Geschichte des Schwurs auf das Evangelium nicht unwillkommen sein. Der Artikel „Eidesrecht" in der $\mathrm{PRE}^{3}$ 5, 248 geht nicht näher auf das Alter dieser Sitte ein, sondern hebt nur hervor: „von altersher wurde es als ein Vorrecht der Geistlichen (später wenigstens der Bischöfe) betrachtet, propositis tantum, sed non tactis evangeliis... die Hand auf die Brust legend (wie auch nach deutschem Brauch Frauenspersonen $z u$ schwören pflegten), ihre Eide $z u$ leisten.

Maulbronn.

Eb. Nestle.

\section{Zu LC 4, 18. x9.}

Im zweiten Jahrgang dieser Zeitschrift habe ich S. 153-I57 gefordert, man solle in der in der Überschrift genannten Stelle wieder zu der früheren Interpunktion und Erklärung zurückkehren:

Der Geist des Herrn ist auf mir, weil er mich gesalbt hat:

Frohes zu verkünden den Armen hat er mich gesandt,

$\mathrm{Zu}$ predigen den Gefangenen Freiheit und den Blinden Gesicht usw. Ich habe zum Schluß insbesondere auch auf eine Stelle aus der Demonstratio des Eusebius hingewiesen, deren Zusammenhang beweise, daß auch Eusebius das ěxpićv $\mu \in$ für sich genommen und nicht, wie gegen- 


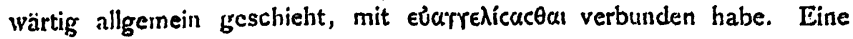
der wichtigsten Stellen des Euscbius war mir damals nicht gegenwärtig, kommt nir aber jetzt in der neuen Ausgabe der Kirchengeschiclite unters Augc. I. 3 druckt jetz.t Schwartz (S. 34, 12): $\pi v \in \hat{O} \mu \alpha$ kupiou $\varepsilon \pi^{\prime \prime} \quad \epsilon \mu \hat{\epsilon}$,

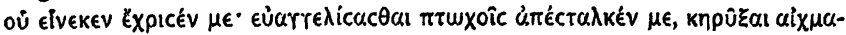
$\lambda$ punction vgl. ecl. proph. 229, 13 ". Die syrische Übersetzung der Euscbianischen Kirchengeschichte hat an dieser Stelle, was anzuführen Schwartz mit Recht unterläbt, in näherem, doch nicht vollständigem Anschluß an die syrische Kirchenbibel, wie aus meiner Verdeutschung zu entnehmen ist: „weil er mich gesalbt hat, daß ich Frohbotschaft bringe den Armen und mich gesandt hat zu verkündigen usw." Wenn ich vor zwei Jahiren schlob: Das Beigebrachte dürfte genügen, eine Revision der Fragc anzuregen, so bin ich heute noch viel entschiedener als schon damals der Überzeugung, dab die alte, hier auch bei Eusebius befolgte Interpunktion im NT die richtige ist. ${ }^{x}$

Maulbronn.

Eb. Nestle.

\section{Joh I, x. 2.}

In allen genauen Ausgaben und Erklärungen wird erörtert, daß die Abtremnung der Sätze zwischen V. 3 und 4 im ersten Kapitel des vierten Evangeliums streitig sei. Man könne den Punkt, wie meistens für richtig gehalten wird, hinter $\delta$ rérovev setzen; man könne diese beiden Worte aber auch als Subjekt des nächsten Satzes betrachten, in welch letzterem Fall wieder eine doppelte Möglichkeit entstehe, das Komma vor oder hinter èv aủrŵ zu setzen. ${ }^{2}$ Dagegen finde ich in keiner der neueren

I Nachschrift bei der Korrektur: Welches Schwanken an dieser Stelle berrscht, dafür noch einige Belege: Stephanus 1550 und Mill 1707 haben die alte Interpunktion; die Lloyd'sche Wiederholung von Mill 1828 die neue, 1836 die alte, 1889 wieder die

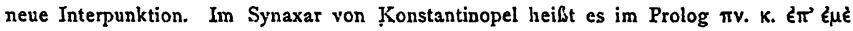

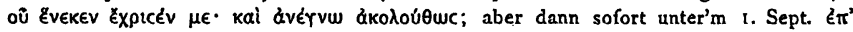

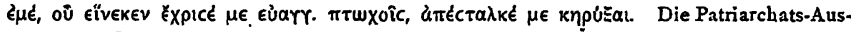
gabe des N. T. von 1903 hat die alte Interpunktion, aber der lateinische Irenäus IV, 37, I (nach der Anführung bei Merx Die vier kanon. Evv. II, 2216 ) super me quapropter unxit me evangelisare pauperibus, misit me curare etc., nach Harvey dagegen: unxit me, ev. pauperibus misit me, curare. Sogar die beiden Päpste Sixtus V. und Clemens VIII. beweisen ihre concordia discors auch an dieser Stelle (s. meine Ausgabe des lat. N. Ts.). Wellhausen vertritt offenbar die alte Fassung; ebenso Epiphanius, haer. 5 I, p. 447.

2 Nur anmerkungsweịse sei darauf hịgewiesen, daß auch dies Év aủ tụ selbst 
Ausgaben oder Erklärungen eine Bemerkung darüber, daß ganz dieselbe Frage auch schon zwischen V. I und 2 entsteht. Zuerst stief. ich darauf in dem Aufsatz von A. N. Jannaris St. John's Gospel and the Logos in Bd. 2 dieser Zeitschrift S. 24, wo er den Eingang des Evan-

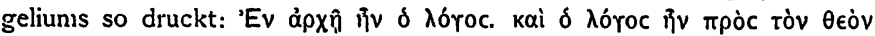

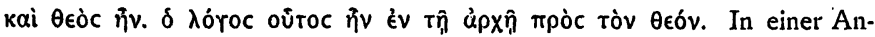
merkung hebt er ausdrücklich hervor: "This is one of the numerous instances of mispunctuation and consequent misinterpretation in the New Testament, especially in St. John." Ich glaubte zunächst es mit einem Sport dieses Verfassers zu tun zu haben, da er sófort in V. 8- io eine älnnliche neue Interpunktion einführt, sehe aber zufällig, daß dieselbe Interpunktion schon vor sehr langer Zeit gemacht worden ist und sogar den Gegenstand dogmatischer Erörterungen gebildet hat. Man lese in den Prolegomena von Mill's Ausgabe von I707 p. LXXIX in dem Abschnitt, der von Ambrosius handelt:

Joan. I. I. In principio erat verbum, \& verbum erat apud Deum, \& deus erat verbum. notat Ambrosius (ut et Hilarius diac. ac Augustin.) a Photino avulsum fuisse verbum, quod est in posteriore versiculi parte, a praecedentibus per interpunctionem, hoc modo, Et Deus erat. Verbum hoc in principio etc. ne scilicet hinc concluderetur Christum esse Deum. Sed quis non videt Christum pariter $\theta \in 0 \lambda$ oreîc $\theta \alpha$, hoc an illo modo verba ista interpungas? Expressa enim Joannis sententia erit, ^órov fuisse ab initio, (seu ab aeterno) fuisse apud Deum, \& fuisse revera Deum. Nisi enim in posteriori hac parte, \& Deus erat, subintelligas Verbum, oratio erit mire absurda; in qua scil. affirmato primùm Christum fuisse Deum, sequatur mox Deum hunc fuisse, seu exstitisse in rerum natura: quod nemo sanus scripserit, multo minus Evangelista $\theta$ єómveuctoc. Hoc cum advertissent Augustinus, (lib. 3 de Doctr. Christ. c. 2.) \& Ambrosiaster (Quaest. V. \& N. T. c. 9I.) \& vero distinctione hac usos notâssent Photinianos quosdam suorum temporum, ad argumenti pro Christi divinitate hinc desumti vim eludendam; eo ducti sunt, ut interpunctionem istam, utcunque commodam satis, Haeresique neutique propitiam, a Photino, eiusque sectatoribus, primam factam crederent.

Ob diese Interpunktion, die Mill sogar für satis commodam erklärt, in irgend einer Handschrift des griechischen Textes oder einer Übersetzung bezeugt ist, weiß ich nicht. Da auch Bengel sie nicht erwähnt,

wieder doppeldeutig ist, ob masculin oder neutrum; so Jannaris an der gleich $2 \mathfrak{u}$ nennenden Stelle. 
der 25 Quartseiten auf die Widerlegung der jetzt vergessenen Konjektur $\theta \in O 0$ für $\theta \in \dot{c} c$ in V. I verwendet, sei auf diesen Vorgänger von Jannaris hingewiesen.

Maulbronn.

Eb. Nestle.

\section{"Epistolae Clementis."}

Dic Korintherbricfe des Clemens sind in Europa griechisch nur hinter dem neutestamentlichen Teil des Codex Alexandrinus, syris $\mathrm{ch}$ nur in der einen jetzt in Cambridge befindlichen Handschrift Julius Mohls zwischen dem Judas- und Römerbrief zugänglich. Zn allgemeiner Überraschung hat Morin 1894 auch einen latcinischen Text entdeckt.

Was mag hinter der Bezeichnung epistolae Clementis stecken, die in folgender Beschreibung der Bibliothek von Toul aus der Zeit des Abts Wido vor 1084 vorkommt? Bei G. Becker, Catalogi Bibliothecarum antiqui p. I49:

1) Pandecten totius divinae legis veteris ac novi testamenti vol. I. 2) Pentateucum Moysy vol.. I. 3) lib. Josuae cum epistolis Clementis vol. I. 4) lib: reg. vol. I. 5) lib. prophetarum vol. I. 6) lib. Salomonis cum Job. vol. I. 7) lib. Tobiae cum libro Nachabeorum vol. I. 8) actus apostolor: cum apocalypsi et VII ${ }^{\text {tem }}$ epistolis canonicis Et epistolis Pauli apost. vol. I.

Wahrscheinlicher ist es ja wohl, daß man hiebei an die epistola(e) s. Clementis papae ad beatum Jacobum apostolum fratrem Domini zu denken hat, die gleich der älteste Katalog, den Becker veröffentlichte, der von S. Vandeville (zwischen 742 u. 747) aufführt. Immerhin wäre es der Mühe wert, der Sache nachzugehen, falls es nicht Lightfoot, der mir nicht zur Hand ist, nicht schon getan hat. Auf jeden Fall ist die Stellung zivischen Josua und Königsbüchern sehr auffallend.

Oder sind es die zwei Briefe an die Jungfrauen, aus denen der Mönch Antonius um 620 reiche Excerpte in seine „Pandekten der

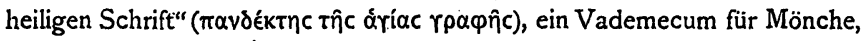
aufnahm? (Bardenhewer, Altkirchliche Literatur I, 114). Auch ihre Auffindung in Latein wäre sehr erwünscht.

Maulbronn.

Eb. Nestle. 